\title{
Ludische Wege zu hochschulrelevanten Zielen - Optimierte Aussprache und Präsentationstechniken durch gerappte und selbst verfasste kreative Texte
}

\author{
Nils Bernstein
}

Die zentralen Thesen des Kurzvortrags in Form eines „Sonnenstrahles“ auf dem Scenario-Symposium lassen sich schnell umreißen. Im Zusammenhang eines Scenario-Symposiums mit vielen Teilnehmenden aus der Fremdsprachendidaktik, die für eine performative Lehr- und Lernkultur an Hochschulen eintreten, rennt man damit womöglich offene Türen ein. Begibt man sich mit diesen Thesen in andere Hochschulbereiche, so ruft man damit wahrscheinlich immer noch Zögern, Stirnrunzeln oder gar Ablehnung hervor - Haltungen also, die es zu widerlegen gilt. Die Thesen lauten:

1. Wer kreative fiktionale Texte selbst schreibt, verbessert seine sprachlichen Leistungen in allen anderen Kontexten.

2. Wer kreative Texte analysiert, der/die kann auch Sachtexte besser analysieren und beschreiben.

3. Werfremdeliterarische Texte szenischinterpretiert oder eigeneliterarische Texte vorträgt, bereitet sich perfekt vor auf sprachliche Performance (zum weiteren Verständnis dieses Begriffes vgl. Fleiner 2016 : 37ff) in Situationen wie Redebeitrag im Seminar, Präsentation, mündliche Prüfung, Bewerbungsgespräch u.ä.

4. Der Grund für vorgenannte These liegt am gezielten Stimmtraining, dem erhöhten Körperbewusstsein, einem sichereren Stand, einem bewussteren Einsatz von Mimik und Gestik, der Reflektion von Selbst- und Fremdwahrnehmung und einem bewussteren Umgang mit Nervosität. (vgl. Stöver-Blahak 2014: 124f.)

Ein Beispiel für eine kreative Sprachleistung, die sich ebenso auf akademische Kontexte anwenden lässt, wäre die Aufgabenstellung, eine Aussage informell zu formulieren, wie man sie etwa in einem vertrauten Kreis äußern würde. Diese Aussage soll dann durch lexikalische und/oder syntaktische Modifikation registerspezifisch modifiziert werden, wie man sie in einer formellen Situation verwenden würde. Eine informelle Formulierung wäre etwa, gemäß einer 
Aufgabenstellung aus dem Lehrwerk Wissenschaftssprache verstehen. Wortschatz - Grammatik - Stil - Lesestrategien: „Also, wenn ich mir meinen Schatz so anschaue, dann muss ich ja schon sagen, dass er eine ganz schöne Wampe hat.“ Die formelle Äußerung lautet: „Bei genauerer Betrachtung meines Lebensgefährten ist festzustellen, dass das Maß seines Bauchumfanges die der Gesundheit förderlichen Werte mittlerweile deutlich übersteigt" (Fügert \& Richter 2015: 37). Studierende des Seminares zu wissenschaftlichem Arbeiten an der Universität Hamburg haben in ihren Leistungen der Bewältigung dieser Aufgabe viel Kreativität an den Tag gelegt und das bildungssprachliche Register in hyperbolischem Maße bedient. Für den Alltagskontext lautete die Aussage: „Weil der Student sich für die Vorlesung nicht interessiert hat, hat er während der Vorlesung einen japanischen Manga gelesen." Daraus wurde: „Mangels Interesse an der Vorlesung warf der Student inzwischen einen Blick auf ein japanisches jugendkulturelles literarisches Werk." In der Tat finden solche Umformulierungen im bildungssprachlichen Kontext andauernd statt. Würde man in vertrautem Kontext äußern: „Wie sich das genau verhält, weiß kein Mensch" so lautete dieselbe Aussage im akademischen Kontext womöglich: „Die Definitionsversuche zur umrissenen Fragestellung sind in der Forschungsdebatte defizitär. “1

Ein vertieftes Textverständnis verspricht auch folgende Aufgabenstellung zum Verfassen kreativer fiktionaler Texte, die im Ursprung von dem Slam-Künstler Dalibor Marković stammt: Zunächst werden gemeinsam mit der Gruppe der Lernenden (literarische und nicht-literarische) Textsorten gesammelt. Darunter können so unterschiedliche Nennungen wie „Gedicht“, „Romananfang“ oder auch „Tweet" und „Busfahrplan“ fallen. Anschließend werden Themen genannt, mit denen man sich oftmals in Literatur auseinandersetzt. Häufige Nennungen sind hier „Liebe“, „Tod“, „Träume“. Und schließlich werden einige einfache Stilmittel gesammelt, beispielsweise „Metapher“, „Reim“, „Alliteration“usw. Die Aufgabe ist es dann, einen zufallsgenerierten Schreibauftrag aus den Nennungen zu erstellen wie etwa: Schreiben Sie einen Tweet über die Liebe, der viele Alliterationen enthält. Folgender Text entstand von einem Deutschlernendem auf dem Niveau C1 mit dem Auftrag, einen wissenschaftlichen Text über das Thema Erwachsenwerden mit vielen Reimen zu schreiben:

„Pubertät ist ein komplexes Thema / und passt in kein festes Schema. / Im vorliegenden Artikel werden wir versuchen, / diesen Zustand zu untersuchen. / [...] Obwohl manche Teenager gemein /sind, sind sie auf 'ner bösen Welt nicht allein. / Die Folge ist simpel und relativ klar / - Probleme der Jugend sind völlig lösbar."

\footnotetext{
${ }^{1}$ Auch wenn die Aussage wahrscheinlich sehr ähnlich ist, irritieren informelle Äußerungen im akademischen Kontext und können zu Missverständnissen führen, wie der Verfasser auf der Scenario-Konferenz am eigenen Leib erfuhr. Nach dem Plenarvortrag von Manfred Schewe fragte er: „Sambanis (2015)“. Was ist das? Die Antwort lautete, damit solle ein Übergang zum Folgevortrag geschaffen werden, denn dieser würde von Sambanis gehalten. Registerspezifisch korrekt und zielführend wäre allerdings die Frage gewesen: „Wie lauten die vollständigen bibliographischen Angaben zu ,Sambanis (2015)‘?“
} 
Erfolgsversprechend ist ein Vortrag im Sprechgesang. Dazu hinterlegt man diesen Text mit einem einfachen Beat aus Bass- und Snare-Drum, jeweils abwechselnd auf der 1 (bass), der 2 (snare), der 3 (bass) und der vier (snare), wobei ein Schnipsen mit der Hand die Achtelnoten die Hi-Hat imitiert. Durch eine solche rhythmische Unterstützung wird deutlich, dass das originelle Enjambement „gemein/sind“ in einem gerappten Vortrag den Reim „gemein... allein“ ermöglicht. Darüber hinaus lässt sich in der gelungenen Bewältigung des Schreibauftrages zeigen, dass die ungewöhnliche Juxtaposition von „wissenschaftlichem Artikel“ und „Reimen“ zur Anwendung und Vertiefung registerspezifischer Redemittel wie im vorliegenden Artikel sowie zur Reflektion bildungssprachlicher Textsortenspezifik führte. Diese erworbene Fertigkeit lässt sich ebenso anwenden auf andere Sprachhandlungen im bildungssprachlichen Bereich wie etwa „berichten, beschreiben, begründen, argumentieren, versprachlichen, modellieren, diskutieren, erläutern, protokollieren, lesen, schreiben" (Leisen 2018:11). Anders gesagt: Wer kreative Texte $\mathrm{zu}$ schreiben und $\mathrm{zu}$ analysieren vermag, ist auch in der Lage bei akademischen Texten wie etwa Hausarbeiten auf Treffsicherheit der Formulierungen zu achten. Eine performativ orientierte Lernumgebung, ein auf Performance orientierter Unterricht wie er beispielsweise mit dem szenischen Interpretieren von (fremden) Gedichten oder aber mit dem Fokus auf Slam Poetry und dem Vortragen selbst geschriebener Texte gegeben ist, berücksichtigt den zentralen Faktor der Lernmotivation, vermittelt bildungssprachliche Inhalte und führt zu besseren Leistungen in allen mündlichen Präsentations- und Gesprächssituationen. Wünschenswert ist daher, dass eine Verankerung szenischen Lehrens und Lernens an Hochschulen erfolgt, um trockene Szenarien, die dem Muster teaching to the test folgen, zu vermeiden und den Unterricht bei gleichzeitiger Berücksichtigung der Lerntypen um den zentralen Faktor der Lernmotivation zu bereichern.

\section{Bibliografie}

Fleiner, Micha (2016): Performancekünste im Hochschulstudium. Transversale Sprach-, Literatur- und Kulturerfahrungen in der fremdsprachlichen Lehrerbildung. Berlin / Strasburg (Um.) / Milow

Fügert, Nadja \& Richter, Ulrike A. (2015): Wissenschaftssprache verstehen. Wortschatz - Grammatik - Stil - Lesestrategien. Stuttgart: Klett

Leisen, Josef (2018): Von der Alltagssprache über die Unterrichtssprache zur Fachsprache. Sprachbildung im Fachunterricht. In: Fremdsprache Deutsch 58, $10-23$

Stöver-Blahak, Anke (2014): Ästhetische Kommunikation im DaF-Unterricht. Ich habe es eher als ein Arbeitswerkzeug betrachtet als ein richtiges Gedicht. In: Bernstein, Nils / Lerchner, Charlotte (Hrsg.): Ästhetisches Lernen im DaF-/DaZ-Unterricht. Literatur - Theater - Bildende Kunst - Musik - Film. Göttingen, 121-133 